\title{
A ROBUST ITERATIVE ALGORITHM FOR WIRELESS MIMO ARRAY AUTO-CALIBRATION
}

\author{
Nathaniel B. Shelton and Brian D. Jeffs \\ Brigham Young University, Department of Electrical and Computer Engineering \\ Provo, UT 84602, email: bjeffs@ee.byu.edu
}

\begin{abstract}
An algorithm is presented for estimating complex transmit and receive array calibration gains in a MIMO communications system. Calibration values are computed from a series of distinct channel transfer function matrix estimates over a time-varying channel. The new method, called RIMM, is an extension of a previous algorithm proposed by Friedlander and Weiss [1]. Several significant weaknesses in the earlier algorithm are demonstrated, and robust solutions are presented. An auto-calibration experiment is presented using $2.43 \mathrm{GHz}$ indoor channel probing data samples.
\end{abstract}

\section{INTRODUCTION}

In multiple-input-multiple-output (MIMO) wireless communications systems it is often desirable to understand properties of the multipath propagation channel between transmit and receive arrays of antennas. A number of researchers have reported on channel probing experiments to characterize MIMO channel statistics, evaluating such physical characteristics as multipath ray structure, angle spread, delay spread, raypath clustering, and array correlation [2][3]. Estimating these channel features requires accurate knowledge of array parameters such as element locations and complex gain responses. This paper presents an improved method for array auto-calibration to estimate these gains using a series of channel transfer matrix estimates $\hat{\mathcal{H}}_{i}$. Though knowledge of $\mathcal{H}$ alone (with unknown calibration gains) is sufficient for MIMO space-time coding communication algorithms, channel probing and physical property analysis require a calibrated array. The proposed algorithm is also applicable in single-sided array DOA estimation problems where it is desirable to perform array auto-calibration using signals of opportunity.

The scenario examined in this paper assumes a MIMO channel where the antenna locations are known, but the antenna complex gains (the array calibration) must be estimated. It will also be assumed that estimates are available for $M$ different channel realizations, $\mathcal{H}_{i}, 1 \leq i \leq M$, all with common transmitter and receiver gains, $\gamma$, and $\kappa$ respectively (see Figure 1 ). This situation might be realized, for example, in a mobile communications application, or in a stationary communications platform where the physical surroundings are changing over time.

This paper presents a MIMO auto-calibration algorithm that is a modification of the iterative MUSIC (IM) method proposed by Friedlander and Weiss [1]. The new Robust Iterative Multiplechannel MUSIC (RIMM) method resolves convergence and bias problems of the original algorithm and extends it to exploit the additional information available in multiple channel realizations.

This project is supported by the National Science Foundation.

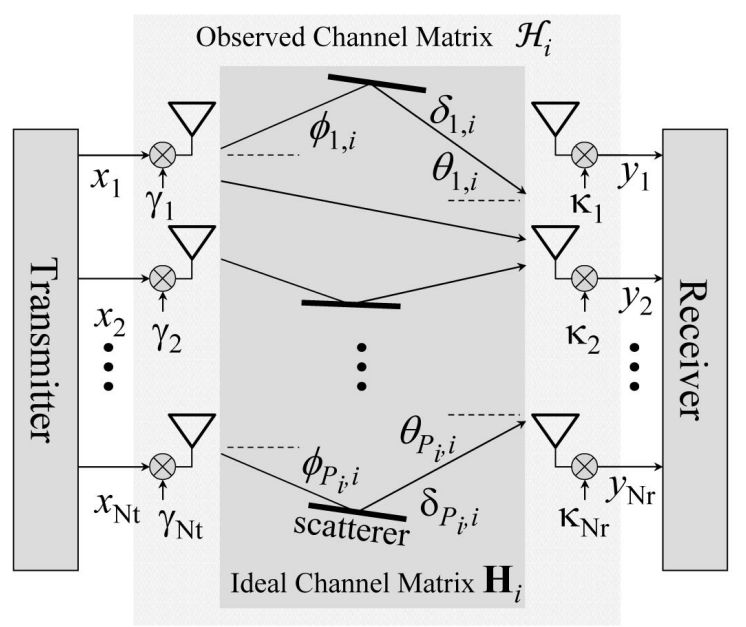

Fig. 1. The ray model for the $i$-th MIMO channel realization, with calibration perturbations on the transmitters and receivers.

The model adopted for the channel is based on ray parameters for the $P_{i}$ propagation paths, as illustrated in Figure 1. Horizontal plane propagation, flat fading and specular scattering are assumed. Thus for the $i$-th channel realization, the $p$-th ray, $1 \leq p \leq P_{i}$, can be identified by an angle for the direction of departure (DOD), $\phi_{p, i}$, an angle for the direction of arrival (DOA), $\theta_{p, i}$, and a complex scalar, $\delta_{p, i}$, for the path amplitude and phase. Identical directional response is assumed for each element across the transmitter array, and separately for the receiver array, so that the respective gains, $\gamma$ or $\kappa$, are independent of direction.

Each observed channel matrix, $\mathcal{H}_{i}=\operatorname{diag}(\boldsymbol{\kappa}) \mathbf{H}_{i} \operatorname{diag}(\boldsymbol{\gamma})$, includes unknown calibration gains and represents the effective linear transfer function from an $N_{t}$-long transmitted vector, $\mathbf{x}$, to the resulting $N_{r}$-long received vector, $\mathbf{y}$, i.e., $\mathbf{y}=\mathcal{H}_{i} \mathbf{x}$. The autocalibration algorithm operates on estimates, $\hat{\mathcal{H}}_{i}$, of the this channel which are typically obtained by probing with a known sequence of training symbols, $\mathbf{x}[n]$, observing $\mathbf{y}[n]$, and solving the inverse problem for $\hat{\mathcal{H}}_{i}$. The ideal (calibrated) channel matrix, $\mathbf{H}_{i}$, is directly related to its ray parameters as follows

$$
\begin{aligned}
\mathbf{H}_{i} & =\mathbf{A}_{r}\left(\boldsymbol{\theta}_{i}\right) \operatorname{diag}\left(\boldsymbol{\delta}_{i}\right) \mathbf{A}_{t}^{H}\left(\boldsymbol{\phi}_{i}\right), \text { where } \\
\mathbf{A}_{r}\left(\boldsymbol{\theta}_{i}\right) & =\left[\mathbf{a}_{r}\left(\theta_{1, i}\right) \ldots \mathbf{a}_{r}\left(\theta_{P_{i}, i}\right)\right] \\
\mathbf{A}_{t}\left(\boldsymbol{\phi}_{i}\right) & =\left[\mathbf{a}_{t}\left(\phi_{1, i}\right) \ldots \mathbf{a}_{t}\left(\phi_{P_{i}, i}\right)\right]
\end{aligned}
$$

and where $\mathbf{a}_{r}(\theta)$ and $\mathbf{a}_{t}(\phi)$ are the array-response vectors for a 
plane wave arriving from angle $\theta$ or $\phi$ respectively for the receivers and transmitters. These response vectors are evaluated at $P_{i}$ DOA angles and $P_{i}$ DOD angles corresponding to the $P_{i}$ rays. For plane-wave signals the response vectors are defined by the array geometry as follows:

$$
\begin{aligned}
\mathbf{a}_{r}(\theta) & =\left[e^{j \frac{\omega}{c} \vec{r}_{1} \cdot \vec{s}_{\theta}}, \ldots, e^{j \frac{\omega}{c} \vec{r}_{N_{r}} \cdot \vec{s}_{\theta}}\right]^{T}, \\
\vec{s}_{\theta} & =\hat{i} \cos \theta+\hat{j} \sin \theta,
\end{aligned}
$$

where $\vec{r}_{l}$ is the 3 -D position vector for the $l$-th element of the receive array, and $\hat{i}$ and $\hat{j}$ are unit vectors along $\mathrm{x}$ and y axes respectively. $\mathbf{a}_{t}(\phi)$ and $\vec{t}_{l}$ are defined similarly.

\section{ROBUST ITERATIVE MULTIPLE-CHANNEL MUSIC}

The problem addressed in this section is as follows: given observations $\hat{\mathcal{H}}_{i}, i=1 \ldots M$, estimate $\kappa$ and $\gamma$. The proposed approach will in the process estimate the $\theta_{p, i}$ 's and $\phi_{p, i}$ 's (though not necessarily their pairings in the $p$-index, i.e. we will not determine which estimated arrival angle belongs to a given departure angle for a single ray) for each channel $\mathcal{H}_{i}$.

As in IM [1], we will use a two-phase iterative procedure to alternately estimate ray angles and calibration gains. This is done by expressing each channel estimate in terms of its partitioned singular value decomposition,

$$
\mathcal{H}_{i}=\left[\mathbf{U}_{s, i} \mid \mathbf{U}_{n, i}\right] \boldsymbol{\Sigma}_{i}\left[\mathbf{V}_{s, i} \mid \mathbf{V}_{n, i}\right]^{H}
$$

where subscript ' $s$ ' indicates singular vectors corresponding to the signal subspace, i.e. those with non-zero singular values in the diagonal $\boldsymbol{\Sigma}_{i}$. Subscript ' $n$ ' indicates the orthogonal subspace of singular vectors corresponding to the zero singular values. When a sample estimate, $\hat{\mathcal{H}}_{i}$, is used in the algorithm described below, $\mathbf{U}_{n, i}$ and $\mathbf{V}_{n, i}$ contain noise induced sample error components, and will therefore be refered to as the 'noise' subspaces.

$\operatorname{Diag}(\boldsymbol{\kappa}) \mathbf{A}_{r}\left(\boldsymbol{\theta}_{i}\right)$ spans the same space as $\mathbf{U}_{s, i}$, and is orthogonal to $\mathbf{U}_{n, i}$. For the single-ended array DOA estimation problem (as opposed to MIMO channel probing) $\mathbf{U}_{n, i}$ would be formed as the partitioned eigen decompositon of sample autocorrelation matrix $\hat{\mathbf{R}}_{y y}$. The following discussion focuses on estimating receiveside parameters $\boldsymbol{\kappa}$ and $\boldsymbol{\theta}_{i}$ from $\hat{\mathbf{U}}_{n, i}$. Due to the symmetry of the problem, developing a corresponding estimate of $\gamma$ and $\phi_{i}$ from $\hat{\mathbf{V}}_{n, i}$ is trivial.

\section{Iteration Phase I}

The first step of the $k$-th RIMM iteration is identical to that of IM. The calibration gain estimate from the immediate past iteration, $\kappa^{k-1}$, is used to form a MUSIC scan for the DOAs:

$$
\begin{aligned}
\boldsymbol{\theta}^{k} & =\arg \operatorname{local} \min _{\theta} V\left(\theta, \boldsymbol{\kappa}^{k-1}\right), \text { where } \\
V(\theta, \boldsymbol{\kappa}) & =\left\|\hat{\mathbf{U}}_{n, i}^{H} \operatorname{diag}(\boldsymbol{\kappa}) \mathbf{a}_{r}(\theta)\right\|_{2},
\end{aligned}
$$

$\|\cdot\|_{2}$ is the vector $l_{2}$ norm, and the angles in $\boldsymbol{\theta}^{k}$ are selected as the $P_{i}$ smallest local minima. Superscripts ' $k$ ' indicate estimated values which are dependent on the iteration number.

Iteration Phase II

In the second step of each iteration, $\theta_{p, i}$ estimates from phase I are used to form a constrained cost function which is minimized to estimate $\kappa$. The constraint is chosen to preclude the trivial solution at $\boldsymbol{\kappa}=\mathbf{0}$. The IM phase II estimate is

$$
\begin{aligned}
\boldsymbol{\kappa}_{\mathrm{IM}}^{k} & =\arg \min _{\boldsymbol{\kappa}}\|\mathbf{v}\|_{2}^{2}, \text { such that } \kappa_{1}=1, \text { where } \\
\mathbf{v} & =\left[V\left(\theta_{1}^{k}, \boldsymbol{\kappa}\right), \ldots, V\left(\theta_{P_{i}}^{k}, \boldsymbol{\kappa}\right)\right]^{T},
\end{aligned}
$$

and $V(\theta, \kappa)$ is defined in (5). The constraint, $\kappa_{1}=1$, fixes the first element of $\boldsymbol{\kappa}$ arbitrarily to enforce $\boldsymbol{\kappa} \neq \mathbf{0}$. This is permissible since the gain vectors are ambiguous to within a constant common factor across all terms. IM exhibits three (at least) problems due to the form of (6) and iteration over a single channel realization: 1) calibration gains are biased low, 2) poor phase I DOA estimates often dominate and lead to irrecoverably bad solutions for $\kappa$, and 3 ) any given single channel realization may not yield convergence to a valid $\kappa$ estimate.

The RIMM algorithm addresses these problems by iterating over multiple distinct channel realizations and using the following modified phase II optimization

$$
\begin{aligned}
\boldsymbol{\kappa}_{\mathrm{RIMM}}^{k} & =\arg \min _{\kappa}\|\mathbf{v}\|_{0.4}^{0.4}, \text { such that }\|\boldsymbol{\kappa}\|_{2}=\sqrt{N_{r}}, \\
\|\mathbf{v}\|_{0.4}^{0.4} & =\sum_{p=1}^{P_{i}} v_{p}^{0.4}
\end{aligned}
$$

where $\|\cdot\|_{0.4}$ is the $l_{0.4}$ vector pseudo norm.

In IM, requiring $\kappa_{1}=1$ does not prevent smaller magnitude gains from being favored for $\kappa_{m}, m=\left\{2, \ldots, N_{r}\right\}$, when minimizing $\|\mathbf{v}\|_{2}^{2}$. This is particularly true when false DOAs are picked up in phase I so that no good $\kappa$ match is available. In RIMM, $\|\boldsymbol{\kappa}\|_{2}=\sqrt{N_{r}}$ constrains the vector length and does not suffer from this problem. Figure 2 illustrates the bias from (6) and the improvement offered by (7). Scatter plots of calibration estimates for 1000 randomly generated complex gain vectors, $\boldsymbol{\kappa}_{m}$. Estimated calibration gains are shown relative to the true values (i.e. $\hat{\kappa}_{m} / \kappa_{m}$ is plotted). The random channel realizations were generated with an accurate cluster-based multipath model [2]. It can be seen that a bias towards zero in the gain magnitudes has been eliminated by using the RIMM constraint.

RIMM also uses a different optimization metric based on an $l_{0.4}$ norm to calculate $\kappa^{k}$ rather than the $l_{2}$ norm in IM. This modification has the effect of making the gain estimate less sensitive to outliers in $V\left(\theta_{p}^{k}, \boldsymbol{\kappa}\right)$, i.e. MUSIC scan minima that deviate from zero significantly. These outliers are often caused by false DOAs that are picked up in phase I. Figure 3 shows an example of how the two algorithms respond to outliers. In both cases the first iteration selects two minima closely corresponding to true DOAs and one false-DOA minima at about $-50^{\circ}$. In minimizing the original cost function, the outlier dominates the $l_{2}$ norm and a gain estimate is favored that lowers the false minima in the second iteration but not the true minima, as shown in Figure 3(a). In the process, the gain estimate degrades from an initial error of $16 \%$ to $43 \%$ after one iteration. Error percentage is calculated as $\epsilon^{k} \times 100 \%$, where

$$
\epsilon^{k}=\min _{\psi}\left\|e^{j \psi} \frac{\boldsymbol{\kappa}^{k}}{\left\|\boldsymbol{\kappa}^{k}\right\|_{2}}-\frac{\boldsymbol{\kappa}_{\text {true }}}{\left\|\boldsymbol{\kappa}_{\text {true }}\right\|_{2}}\right\|_{2},
$$

and where $\psi$ is adjusted to provide the best match to the arbitrary phase term. When the new cost function is minimized, as in Figure $3(\mathrm{~b})$, the $l_{0.4}$ norm causes a gain estimate to be favored which lowers the true minima in the second iteration. Starting from the same initialization, this gain estimate has an error of only $4 \%$ after the first iteration.

\section{Using Multiple Channel Realizations}

When multiple distinct channel realizations are available it is possible to dramatically reduce the problem (mentioned above) of poor convergence for some individual channels. We propose an algorithm in which the channel index, $i$, is incremented between iterations. Thus the algorithm proceeds in a round through the available channels. Also, the gain estimates are exponentially averaged 
(a)

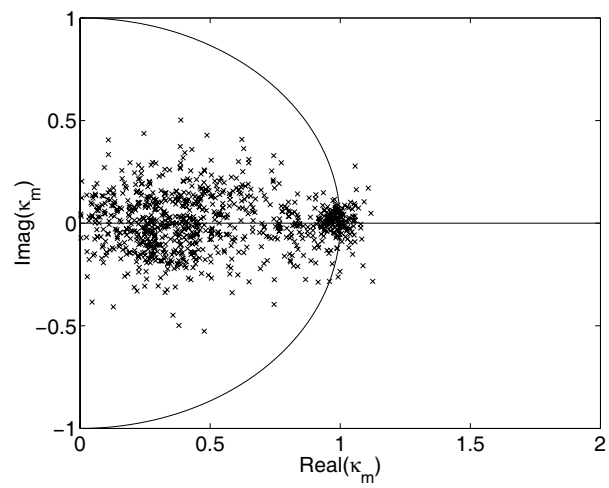

(b)

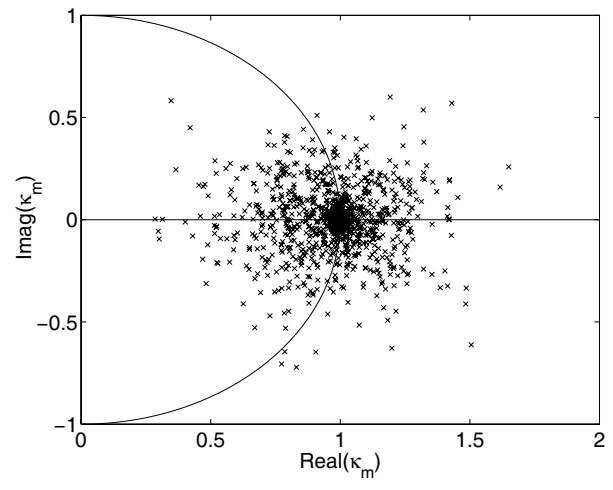

Fig. 2. One thousand estimated calibration gains from simulations, relative to the true calibration gains. (a) Using the original constraint. (b) Using the RIMM, constant-norm constraint.

over the iterations to prevent large deviations due to channels that result in poor estimates. The new algorithm is as follows.

\section{RIMM Algorithm Steps:}

1. Initialization: form noise subspaces $\hat{\mathbf{U}}_{n, i}$ for each channel $i$; set $k=1, i=1$, and $\kappa^{0}=[1,1, \ldots, 1]^{T}$.

2. Find $\boldsymbol{\theta}^{k}$ from the $P_{i}$ lowest minima of (5).

3. Find the iteration gains estimate, $\kappa_{\text {RIMM }}^{k}$, by minimizing (7). This can be computed by gradient descent steps, alternated with normalization to impose the constraint.

4. Use exponential averaging to compute the new smoothed estimate as

$$
\begin{aligned}
\overline{\boldsymbol{\kappa}}^{k} & =(1-\alpha) \overline{\boldsymbol{\kappa}}^{k-1}+\alpha e^{i \psi} \boldsymbol{\kappa}_{\mathrm{RIMM}}^{k}, \\
\psi & =\arg \min _{\psi}\left\|e^{i \psi} \boldsymbol{\kappa}_{\mathrm{RIMM}}^{k}-\overline{\boldsymbol{\kappa}}^{k-1}\right\|_{2} .
\end{aligned}
$$

Note that a best-fit phase rotation is carried out to insure successive add with coherent phase.

5. Normalize the averaged estimate as $\overline{\boldsymbol{\kappa}}^{k}=\overline{\boldsymbol{\kappa}}^{k} \sqrt{N_{r}} /\left\|\overline{\boldsymbol{\kappa}}^{k}\right\|_{2}$.

6. Increment $k$ and $i_{\text {(модицо } M \text { ) }}$ and repeat from step 2. Return $\hat{\boldsymbol{\kappa}}=\overline{\boldsymbol{\kappa}}^{k}$ at convergence.

Figure 4 shows the effect of exponentially averaging the calibration estimate. The "iteration estimate" curve uses no averaging, but computes $\kappa^{k}$ as a separate full IM estimate at each channel (a)

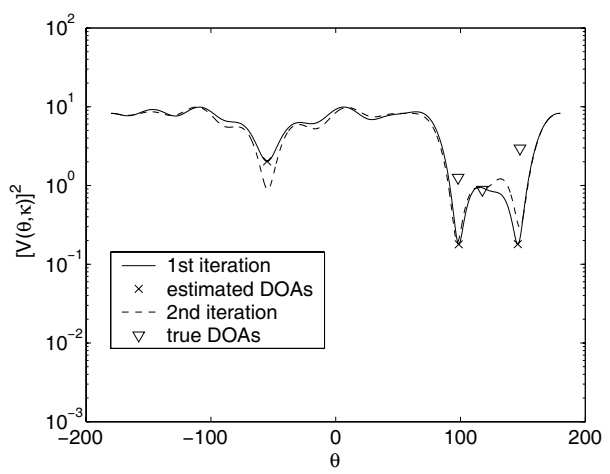

(b)

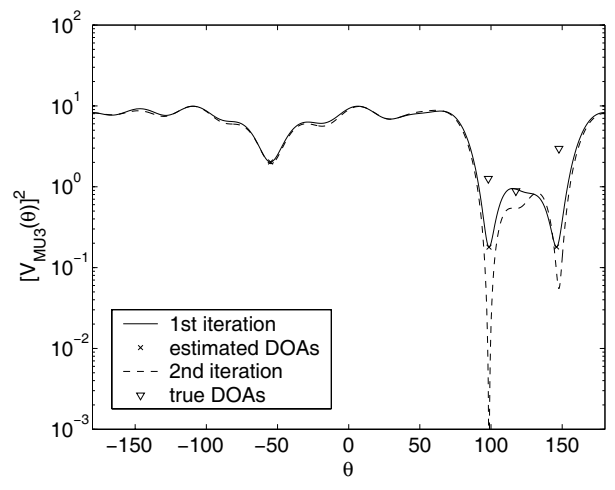

Fig. 3. MUSIC scans from iterative MUSIC in the first and second iterations using the original cost function(a), and using the new RIMM cost function (b).

iteration using the previous channel's result as an initialization. Note the poor convergence behavior. The exponentially averaged RIMM error in $\overline{\boldsymbol{\kappa}}^{k}$ is much lower at convergence.

\section{REAL DATA RESULTS}

The new algorithm was tested on real $2.43 \mathrm{GHz}$ measurement data, using a custom channel probing system built in the Wireless Communications Laboratory at Brigham Young University. The transmitter and receiver arrays each consist of ten monopole antennas in a circular configuration, with a adjacent element spacing of $0.46 \lambda$ and a total aperture of $1.7 \lambda$. See [3] for a more detailed description of the measurement platform.

In [4], results were reported from measurements taken on the fourth floor of the Engineering building on the BYU campus, and the same measurements are used here. The transmitter remained stationary in one hallway while the receiver was moved down an intersecting hallway, as illustrated in Figure 5. Fifty channel estimates were chosen corresponding to a line-of-sight orientation between the transmitter and the receiver, to ensure a fairly strong signal that would be dominated by a relatively small number of rays. Orthogonal training sequences, $\mathbf{x}[n]$, were transmitted to produce a new channel estimate, $\mathcal{H}_{i}$, for each receiver location. Receiver sites were separated by about one wavelength between probing realizations, and channel estimates were then arranged in a random order.

Channel estimate error was found to have an element-wise 


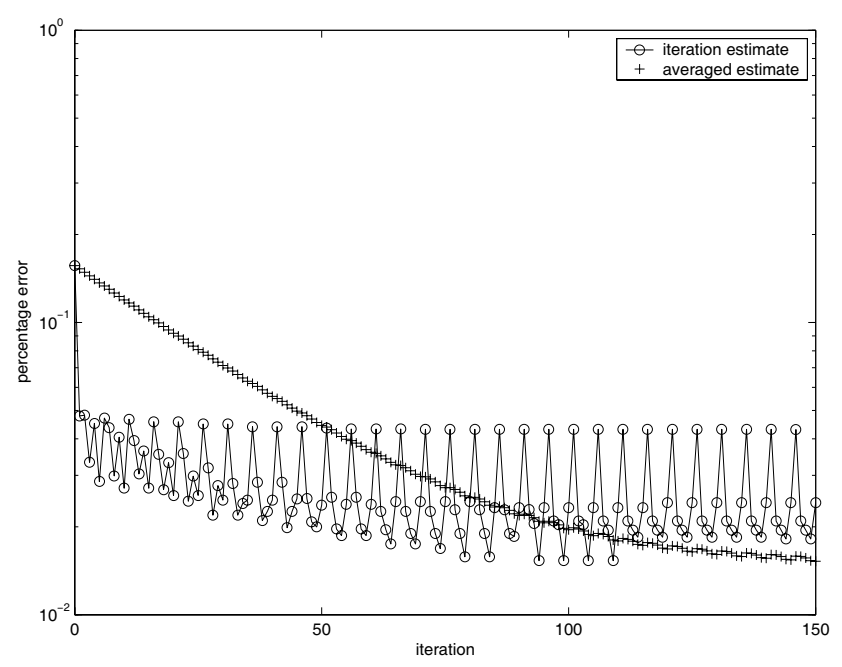

Fig. 4. The convergence of the gains estimate error using the new algorithm. The percentage error is plotted for the iteration estimate, $\boldsymbol{\kappa}^{k}$, and for the averaged estimate, $\overline{\boldsymbol{\kappa}}^{k}$.

variance of about $\sigma_{\mathcal{E}}^{2}=0.00025$ when the transfer matrix was normalized such that $\left\|\hat{\mathcal{H}}_{i}\right\|_{F}=\sqrt{N_{r} N_{t}}$. In addition to this error, we also expect a certain amount of model mismatch. For the 2.43 GHZ indoor propagation environment, diffuse and near-field scatters, along with vertical spread in multipath angle will all likely be present. These factors are not considered in the model, and lead to an environment in which the auto-calibration procedure must be very robust to sources of error.

Since the exact true calibration gains for the test arrays are unknown, the accuracy of the new algorithm was evaluated using a known, multiplicative perturbation inserted on the receiver side of each channel estimate, i.e.

$$
\hat{\mathcal{H}}_{i}^{\prime}=\operatorname{diag}\left(\boldsymbol{\kappa}_{\text {pert }}\right) \hat{\mathcal{H}}_{i}, 1 \leq i \leq M
$$

The i.i.d. complex circular Gaussian random vector, $\kappa_{\text {pert }}$, was generated with mean a of 1 and standard deviation 0.17 . With this known gain offset, the accuracy of the algorithm in tracking the perturbation can be measured by comparing pre- and postperturbation calibration estimates. If the receiver-calibration estimate from the unmodified channel measurements is $\hat{\kappa}$, then the expected estimate from the manually-perturbed channels is $\kappa^{\prime}=$ $\operatorname{diag}\left(\boldsymbol{\kappa}_{\text {pert }}\right) \hat{\boldsymbol{\kappa}}$. We can then compare the actual result, $\hat{\boldsymbol{\kappa}}^{\prime}$, of the algorithm operating on the manually-perturbed channels, $\hat{\mathcal{H}}_{i}^{\prime}$, with this true perturbed calibration, $\boldsymbol{\kappa}^{\prime}$. Tracking error for this estimate is computed using (8) by substituting $\boldsymbol{\kappa}^{\prime}$ for $\boldsymbol{\kappa}_{\text {true }}$, and $\hat{\boldsymbol{\kappa}}^{\prime}$ for $\boldsymbol{\kappa}^{k}$.

Figure 6 plots $\epsilon_{\text {track }}^{k}$ vs. iteration number in for the manuallyoffset real data. The auto-calibration algorithm is able to track the inserted calibration with about 7.3 percent error. These results show reasonable performance, considering the multiple sources of error that are present from model mismatch.

Given the comparative results shown in Figures 2, 3, 4 and 6, we conclude that RIMM is a viable algorithm for MIMO array auto-calibration which significantly outperforms IM.

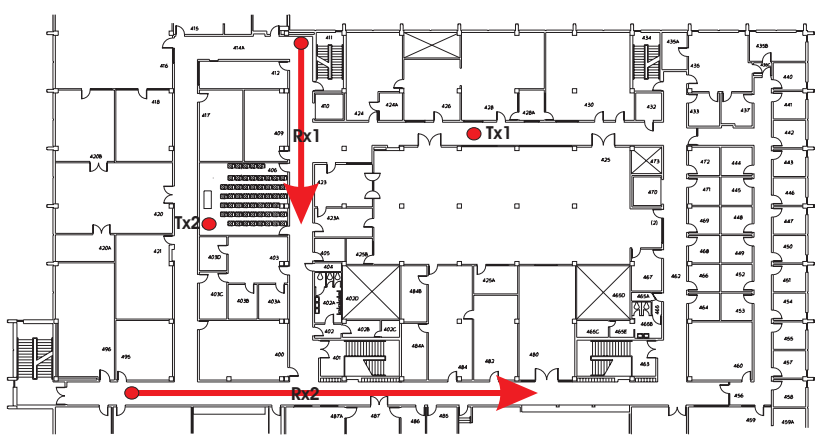

Fig. 5. The fourth floor of the Clyde building, showing the channel environment for the real data measurements and orientations between the transmitter and receiver. The measurements used in this paper correspond to the line-of-sight portion of the position labeled 'Tx1' and 'Rx1'.

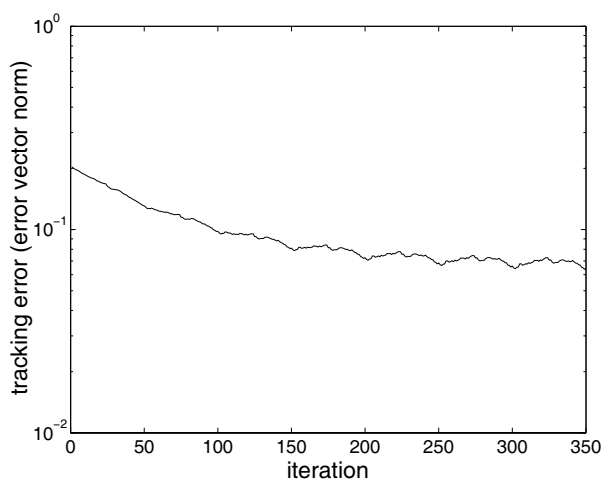

Fig. 6. Tracking error for an manually-injected offset in the receiver calibration for measured data, over the iterations of the autocalibration algorithm.

\section{REFERENCES}

[1] B. Friedlander and A. J. Weiss, "Eigenstructure methods for direction finding with sensor gain and phase uncertainty," in Proceedings of the International Conference on Acoustics, Speech, and Signal Processing, New York, NY, April 1988, pp. 2681-2684.

[2] Q. H. Spencer, B. D. Jeffs, M. A. Jensen, and A. L. Swindlehurst, "Modeling the statistical time and angle of arrival characteristics of an indoor multipath channel," IEEE Journal on Selected Areas in Communications, vol. 18, pp. 347-360, March 2000.

[3] J. W. Wallace and M. A. Jensen, "Measured characteristics of the mimo wireless channel," in Proceedings of the IEEE 54th Vehicular Technology Conference, Atlantic City, NJ, October 2001, pp. 2038-2042.

[4] T. Svantesson and J. Wallace, "Statistical characterization of the indoor mimo channel based on los/nlos measurements," in Conference Record of the Thirty-Sixth Asilomar Conference on Signals, Systems and Computers, November 2002, vol. 2, pp. $1354-1358$. 